\title{
A note on congruence properties of the generalized bi-periodic Horadam sequence
}

\author{
Elif $\operatorname{Tan}^{* 1}$ (D), Ho-Hon Leung ${ }^{2}$ (D) \\ ${ }^{1}$ Department of Mathematics, Ankara University, Ankara, Turkey \\ ${ }^{2}$ Department of Mathematical Sciences, UAEU, Al-Ain, United Arab Emirates
}

\begin{abstract}
In this paper, we consider a generalization of Horadam sequence $\left\{w_{n}\right\}$ which is defined by the recurrence $w_{n}=a w_{n-1}+c w_{n-2}$, if $n$ is even, $w_{n}=b w_{n-1}+c w_{n-2}$, if $n$ is odd with arbitrary initial conditions $w_{0}, w_{1}$ and nonzero real numbers $a, b$, and $c$. We investigate some congruence properties of the generalized Horadam sequence $\left\{w_{n}\right\}$.
\end{abstract}

Mathematics Subject Classification (2010). 11B39, 05A15

Keywords. Horadam sequence, generalized Fibonacci sequence, generalized Lucas sequence, congruence

\section{Introduction}

The generalized bi-periodic Horadam sequence $\left\{w_{n}\right\}$ is defined by the recurrence relation

$$
w_{n}=\left\{\begin{array}{ll}
a w_{n-1}+c w_{n-2}, & \text { if } n \text { is even } \\
b w_{n-1}+c w_{n-2}, & \text { if } n \text { is odd }
\end{array}, n \geq 2\right.
$$

with arbitrary initial conditions $w_{0}, w_{1}$ and nonzero real numbers $a, b$ and $c$. It is emerged as a generalization of the best known sequences in the literature, such as the Horadam sequence, the Fibonacci\&Lucas sequence, the $k$-Fibonacci\& $k$-Lucas sequence, the Pell\&PellLucas sequence, the Jacobsthal\& Jacobsthal-Lucas sequence, etc. Similar to the notation of the classical Horadam sequence [4], we write $\left\{w_{n}\right\}:=\left\{w_{n}\left(w_{0}, w_{1} ; a, b, c\right)\right\}$. In particular, using this notation, we define $\left\{u_{n}\right\}=\left\{w_{n}(0,1 ; a, b, c)\right\}$ and $\left\{v_{n}\right\}=\left\{w_{n}(2, b ; a, b, c)\right\}$ as the generalized bi-periodic Fibonacci sequence and the generalized bi-periodic Lucas sequence, respectively. For the basic properties of the generalized bi-periodic Horadam sequence and some special cases of this sequence, see $[1,3,7,9,11-16]$.

On the other hand, it is important to investigate the congruence properties of different integer sequences. Several methods can be applied to produce identities for the Fibonacci and Lucas sequences. For example, Carlitz and Ferns [2] used polynomial identities in conjunction with the Binet formula to generate new identities for these sequences. The method of Carlitz and Ferns was used by several authors to obtain analogous results for the generalized Fibonacci and Lucas sequences, see $[6,19]$. On the other hand, Keskin and Siar [10] obtained some number theoretic properties of the generalized Fibonacci and Lucas numbers by using matrix method. Moreover, Hsu and Maosen [5] and Zhang [18] applied

\footnotetext{
*Corresponding Author.

Email addresses: etan@ankara.edu.tr (E. Tan), hohon.leung@uaeu.ac.ae (H.H. Leung)

Received: 14.03.2019; Accepted: 04.04.2020
} 
an operator method to establish some of these properties. Recently, Yang and Zhang [17] have studied some congruence relations for the bi-periodic Fibonacci and Lucas sequences by using operator method. But the results [17, Theorem 4.3-4.13] that are obtained by the operator method are incorrect. In this study, by using the method of Carlitz and Ferns [2], we give more general identities involving the generalized bi-periodic Horadam sequences and derive some congruence properties of the generalized bi-periodic Horadam numbers. We collect our main results in Theorem 3.1, Theorem 3.6, and Theorem 3.12. In particular, Theorem 3.1 includes the corrected version of the results given in [17, Theorem 4.7, Theorem 4.9, Theorem 4.11, Theorem 4.13], and Theorem 3.6 includes the corrected version of the results given in [17, Theorem 4.3, Theorem 4.5 ]. Elegant multinomial identities are given in Theorem 3.12. We also obtain a new relation for the generalized bi-periodic Horadam numbers in Theorem 3.11.

The outline of this paper as follows: In Section 2, we give some basic properties of the generalized bi-periodic Horadam sequence. In Section 3, we give some binomial identities and congruence relations for the generalized bi-periodic Horadam sequence by using the method of Carlitz and Ferns [2].

\section{Some preliminary results for the sequence $\left\{w_{n}\right\}$}

In this section, we give some basic properties of the generalized bi-periodic Horadam sequences. To this end, first we recall some properties of the sequence $\left\{u_{n}\right\}$.

The Binet formula of the sequence $\left\{u_{n}\right\}$ is

$$
u_{n}=\frac{a^{\xi(n+1)}}{(a b)^{\left\lfloor\frac{n}{2}\right\rfloor}}\left(\frac{\alpha^{n}-\beta^{n}}{\alpha-\beta}\right)
$$

which can be obtained from [16, Theorem 8]. Here $\alpha$ and $\beta$ are the roots of the polynomial $x^{2}-a b x-a b c$, that is, $\alpha=\frac{a b+\sqrt{a^{2} b^{2}+4 a b c}}{2}$ and $\beta=\frac{a b-\sqrt{a^{2} b^{2}+4 a b c}}{2}$, and $\xi(n)=n-2\left\lfloor\frac{n}{2}\right\rfloor$ is the parity function, i.e., $\xi(n)=0$ when $n$ is even and $\xi(n)=1$ when $n$ is odd. Let assume $\Delta:=a^{2} b^{2}+4 a b c \neq 0$. Also we have $\alpha+\beta=a b, \alpha-\beta=\sqrt{a^{2} b^{2}+4 a b c}$ and $\alpha \beta=-a b c$.

A relation between the sequences $\left\{w_{n}\right\}$ and $\left\{u_{n}\right\}$ can be given in the following lemma.

Lemma 2.1. For any integer $n>0$, we have

$$
w_{n}=u_{n} w_{1}+c\left(\frac{b}{a}\right)^{\xi(n)} u_{n-1} w_{0}
$$

Proof. It can easily be proven by induction.

If we take $c=1$ in Lemma 2.1, it gives the result in [3, Theorem 8].

Now we give the Binet formula of the sequence $\left\{w_{n}\right\}$. We note that the extended Binet formula for the general case of this sequence was given in [7, Theorem 9]. But here we express it in a different manner; that is, our $\alpha$ and $\beta$ are different from the roots which are used in [7].

Theorem 2.2. (Binet Formula) For $n>0$, we have

$$
w_{n}=\frac{a^{\xi(n+1)}}{(a b)^{\left\lfloor\frac{n}{2}\right\rfloor}}\left(A \alpha^{n}-B \beta^{n}\right),
$$

where $A:=\frac{w_{1}-\frac{\beta}{a} w_{0}}{\alpha-\beta}$ and $B:=\frac{w_{1}-\frac{\alpha}{a} w_{0}}{\alpha-\beta}$. 
Proof. By using Lemma 2.1 and the Binet formula of $\left\{u_{n}\right\}$ in (2.1), we have

$$
\begin{aligned}
w_{n} & =u_{n} w_{1}+c\left(\frac{b}{a}\right)^{\xi(n)} u_{n-1} w_{0} \\
& =\frac{a^{\xi(n+1)}}{(a b)^{\left\lfloor\frac{n}{2}\right\rfloor}}\left(\frac{\alpha^{n}-\beta^{n}}{\alpha-\beta}\right) w_{1}+\frac{c b^{\xi(n)}}{(a b)^{\left\lfloor\frac{n-1}{2}\right\rfloor}}\left(\frac{\alpha^{n-1}-\beta^{n-1}}{\alpha-\beta}\right) w_{0} .
\end{aligned}
$$

If $n$ is even, we have

$$
w_{n}=\frac{a}{(a b)^{\frac{n}{2}}}\left(\left(\frac{\alpha^{n}-\beta^{n}}{\alpha-\beta}\right) w_{1}+b c\left(\frac{\alpha^{n-1}-\beta^{n-1}}{\alpha-\beta}\right) w_{0}\right) .
$$

If $n$ is odd, we have

$$
w_{n}=\frac{1}{(a b)^{\frac{n-1}{2}}}\left(\left(\frac{\alpha^{n}-\beta^{n}}{\alpha-\beta}\right) w_{1}+b c\left(\frac{\alpha^{n-1}-\beta^{n-1}}{\alpha-\beta}\right) w_{0}\right) .
$$

From (2.2) and (2.3), we have

$$
\begin{aligned}
w_{n} & =\frac{a^{\xi(n+1)}}{(a b)^{\left\lfloor\frac{n}{2}\right\rfloor}}\left(\left(\frac{\alpha^{n}-\beta^{n}}{\alpha-\beta}\right) w_{1}+b c\left(\frac{\alpha^{n-1}-\beta^{n-1}}{\alpha-\beta}\right) w_{0}\right) \\
& =\frac{a^{\xi(n+1)}}{(a b)^{\left\lfloor\frac{n}{2}\right\rfloor}}\left(\left(\frac{\left(\alpha^{n} w_{1}+b c \alpha^{n-1} w_{0}\right)}{\alpha-\beta}\right)-\left(\frac{\beta^{n} w_{1}+b c \beta^{n-1} w_{0}}{\alpha-\beta}\right)\right) \\
& =\frac{a^{\xi(n+1)}}{(a b)^{\left\lfloor\frac{n}{2}\right\rfloor}}\left(\left(\frac{w_{1}+\frac{b c}{\alpha} w_{0}}{\alpha-\beta}\right) \alpha^{n}-\left(\frac{w_{1}+\frac{b c}{\beta} w_{0}}{\alpha-\beta}\right) \beta^{n}\right) .
\end{aligned}
$$

Since $\alpha \beta=-a b c$, we get the desired result.

By taking initial conditions $w_{0}=2, w_{1}=b$ in Theorem 2.2, we have

$$
v_{n}=\frac{a^{1-\xi(n)}}{(a b)^{\left\lfloor\frac{n}{2}\right\rfloor}}\left(\frac{a b-2 \beta}{a(\alpha-\beta)} \alpha^{n}-\frac{a b-2 \alpha}{a(\alpha-\beta)} \beta^{n}\right),
$$

and by using the relation $\alpha+\beta=a b$, we obtain the Binet formula for the sequence $\left\{v_{n}\right\}$ as follows:

$$
v_{n}=\frac{a^{-\xi(n)}}{(a b)^{\left\lfloor\frac{n}{2}\right\rfloor}}\left(\alpha^{n}+\beta^{n}\right) .
$$

Also by using Lemma 2.1 , we have $v_{n}=b u_{n}+2 c\left(\frac{b}{a}\right)^{\xi(n)} u_{n-1}$. Thus we get a relation between the generalized bi-periodic Fibonacci and the generalized bi-periodic Lucas numbers as:

$$
v_{n}=\left(\frac{b}{a}\right)^{\xi(n)}\left(u_{n+1}+c u_{n-1}\right) .
$$

It should be noted that the generalized Lucas sequence $\left\{t_{n}\right\}$ in [17] is a special case of the generalized bi-periodic Horadam sequence. That is, $\left\{t_{n}\right\}=\left\{w_{n}(2 a, a b ; a, b, 1)\right\}$.

The generating function of the sequence $\left\{w_{n}\right\}$ is

$$
G(x)=\frac{w_{0}+w_{1} x+\left(a w_{1}-(a b+c) w_{0}\right) x^{2}+c\left(b w_{0}-w_{1}\right) x^{3}}{1-(a b+2 c) x^{2}+c^{2} x^{4}},
$$

which can be obtained by taking $r=2$ in [7, Theorem 6].

Also we need the following identity which can be found in [15]:

$$
u_{m n+r}=\frac{a^{1-\xi(m n+r)}}{(a b)^{\left\lfloor\frac{m n+r}{2}\right\rfloor}} \sum_{i=0}^{n}\left(\begin{array}{c}
n \\
i
\end{array}\right) c^{n-i} u_{m}^{i} u_{m-1}^{n-i} u_{i+r} \delta[m, n, r, i]
$$

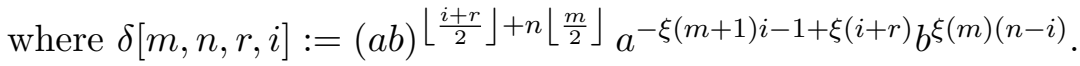




\section{Main results}

In this section, we give some binomial and multinomial identities for the generalized bi-periodic Horadam sequence. As a consequence of these identities, we derive some congruence relations for the sequence $\left\{w_{n}\right\}$. Let $a, b$ and $c$ are positive integers.

Theorem 3.1. For any nonnegative integers $n, r$ and $m$ with $m>1$, we have

$$
w_{m n+r}=\frac{a^{1-\xi(m n+r)}}{(a b)^{\left\lfloor\frac{m n+r}{2}\right\rfloor}} \sum_{i=0}^{n}\left(\begin{array}{c}
n \\
i
\end{array}\right) c^{n-i} u_{m}^{i} u_{m-1}^{n-i} w_{i+r} \delta[m, n, r, i]
$$

where $\delta[m, n, r, i]:=(a b)^{\left\lfloor\frac{i+r}{2}\right\rfloor+n\left\lfloor\frac{m}{2}\right\rfloor} a^{-\xi(m+1) i-1+\xi(i+r)} b^{\xi(m)(n-i)}$.

Proof. Similar to the relation $\gamma^{n}=\gamma F_{n}+F_{n-1}$ for the classical Fibonacci numbers, where $\gamma$ is one of the root of the equation $x^{2}-x-1=0$, we have

$$
\alpha^{m}=a^{-1} a^{\frac{m+\xi(m)}{2}} b^{\frac{m-\xi(m)}{2}} \alpha u_{m}+c a^{\frac{m-\xi(m)}{2}} b^{\frac{m+\xi(m)}{2}} u_{m-1}
$$

and

$$
\beta^{m}=a^{-1} a^{\frac{m+\xi(m)}{2}} b^{\frac{m-\xi(m)}{2}} \beta u_{m}+c a^{\frac{m-\xi(m)}{2}} b^{\frac{m+\xi(m)}{2}} u_{m-1} .
$$

By using the binomial theorem, we have

$$
\begin{aligned}
& \alpha^{m n}=\sum_{i=0}^{n}\left(\begin{array}{c}
n \\
i
\end{array}\right) a^{-i} a^{i \frac{m+\xi(m)}{2}+(n-i) \frac{m-\xi(m)}{2}} b^{i \frac{m-\xi(m)}{2}+(n-i) \frac{m+\xi(m)}{2}} c^{n-i} u_{m}^{i} u_{m-1}^{n-i} \alpha^{i}, \\
& \beta^{m n}=\sum_{i=0}^{n}\left(\begin{array}{c}
n \\
i
\end{array}\right) a^{-i} a^{i \frac{m+\xi(m)}{2}+(n-i) \frac{m-\xi(m)}{2}} b^{i \frac{m-\xi(m)}{2}+(n-i) \frac{m+\xi(m)}{2}} c^{n-i} u_{m}^{i} u_{m-1}^{n-i} \beta^{i} .
\end{aligned}
$$

Multiplying both sides of the above equalities by $A \alpha^{r}$ and $B \beta^{r}$, respectively, and using the Binet formula of $\left\{w_{n}\right\}$, we get

$$
\begin{aligned}
& \left(A \alpha^{m n+r}-B \beta^{m n+r}\right) \\
= & a^{-\xi(m n+r+1)}(a b)^{\left\lfloor\frac{m n+r}{2}\right\rfloor} w_{m n+r} \\
= & \sum_{i=0}^{n}\left(\begin{array}{c}
n \\
i
\end{array}\right)(a b)^{\left\lfloor\frac{i+r}{2}\right\rfloor+n\left\lfloor\frac{m}{2}\right\rfloor} a^{-\xi(m+1) i-1+\xi(i+r)} b^{\xi(m)(n-i)} c^{n-i} u_{m}^{i} u_{m-1}^{n-i} w_{i+r} .
\end{aligned}
$$

which gives the desired result.

We note that it can also be obtained by using Lemma 2.1 and the identity (2.7) as:

$$
\begin{aligned}
& w_{m n+r} \\
& =w_{1} \frac{a^{1-\xi(m n+r)}}{(a b)^{\left\lfloor\frac{m n+r}{2}\right\rfloor}} \sum_{i=0}^{n}\left(\begin{array}{l}
n \\
i
\end{array}\right) c^{n-i} u_{m}^{i} u_{m-1}^{n-i} u_{i+r} \delta[m, n, r, i] \\
& +w_{0} c\left(\frac{b}{a}\right)^{\xi(m n+r)} \frac{a^{1-\xi(m n+r-1)}}{(a b)^{\left\lfloor\frac{m n+r-1}{2}\right\rfloor}} \sum_{i=0}^{n}\left(\begin{array}{c}
n \\
i
\end{array}\right) c^{n-i} u_{m}^{i} u_{m-1}^{n-i} u_{i+r-1} \delta[m, n, r-1, i] \\
& =\frac{a^{1-\xi(m n+r)}}{(a b)^{\left\lfloor\frac{m n+r}{2}\right\rfloor}} \sum_{i=0}^{n}\left(\begin{array}{c}
n \\
i
\end{array}\right) c^{n-i} u_{m}^{i} u_{m-1}^{n-i} \delta[m, n, r, i]\left(w_{1} u_{i+r}+c w_{0}\left(\frac{b}{a}\right)^{\xi(i+r)} u_{i+r-1}\right) \\
& =\frac{a^{1-\xi(m n+r)}}{(a b)^{\left\lfloor\frac{m n+r}{2}\right\rfloor}} \sum_{i=0}^{n}\left(\begin{array}{c}
n \\
i
\end{array}\right) c^{n-i} u_{m}^{i} u_{m-1}^{n-i} w_{i+r} \delta[m, n, r, i] .
\end{aligned}
$$


Remark 3.2. Let $c=1$ in Theorem 3.1. Then we have the following results:

(1) For $m=2 s$ and $r=2 k$, we have

$$
w_{2 s n+2 k}=\sum_{i=0}^{n}\left(\begin{array}{c}
n \\
i
\end{array}\right)\left(\frac{b}{a}\right)^{\frac{i-\xi(i)}{2}} q_{2 s}^{i} q_{2 s-1}^{n-i} w_{i+2 k} .
$$

(2) For $m=2 s$ and $r=2 k+1$, we have

$$
w_{2 s n+2 k+1}=\sum_{i=0}^{n}\left(\begin{array}{c}
n \\
i
\end{array}\right)\left(\frac{b}{a}\right)^{\frac{i+\xi(i)}{2}} q_{2 s}^{i} q_{2 s-1}^{n-i} w_{i+2 k+1} .
$$

(3) For $m=2 s+1$ and $r=2 k$, we have

$$
w_{(2 s+1) n+2 k}=\sum_{i=0}^{n}\left(\begin{array}{c}
n \\
i
\end{array}\right)\left(\frac{b}{a}\right)^{\frac{-i-\xi(i)+n+\xi(n)}{2}} q_{2 s+1}^{i} q_{2 s}^{n-i} w_{i+2 k} .
$$

(4) For $m=2 s+1$ and $r=2 k+1$, we have

$$
w_{(2 s+1) n+2 k+1}=\sum_{i=0}^{n}\left(\begin{array}{c}
n \\
i
\end{array}\right)\left(\frac{b}{a}\right)^{\frac{-i+\xi(i)+n-\xi(n)}{2}} q_{2 s+1}^{i} q_{2 s}^{n-i} w_{i+2 k+1} .
$$

We should note that the above results are the corrected results which are given in [17, Equations (21), (24), (27), (30)], respectively. In particular, see the following example which shows that the results in [17, Equations (21), (24), (27), (30)] do not hold.

Example 3.3. Consider the sequence $\left\{q_{n}\right\}=\left\{w_{n}(0,1 ; 2,1,1)\right\}$. From the recurrence relation of $\left\{q_{n}\right\}$, the terms of the sequence are

\begin{tabular}{c|c|c|c|c|c|c|c|c|c|c|c|c|c|c|c|l}
$n$ & 1 & 2 & 3 & 4 & 5 & 6 & 7 & 8 & 9 & 10 & 11 & 12 & 13 & 14 & 15 & $\ldots$ \\
\hline$q_{n}$ & 1 & 2 & 3 & 8 & 11 & 30 & 41 & 112 & 153 & 418 & 571 & 1560 & 2131 & 5822 & 7953 & $\ldots$
\end{tabular}

If we take $n=2, m=2, k=1$ in [17, Equation (21)], the right hand side of the equation gives

$$
\sum_{i=0}^{n}\left(\begin{array}{c}
n \\
i
\end{array}\right) q_{2 m}^{i} q_{2 m-1}^{n-i} q_{i+2 k}=\sum_{i=0}^{2}\left(\begin{array}{c}
2 \\
i
\end{array}\right) q_{4}^{i} q_{3}^{2-i} q_{i+2}=q_{3}^{2} q_{2}+2 q_{3}^{2} q_{4}+q_{4}^{3}=674,
$$

which contradicts $q_{10}=418$.

If we take $n=2, m=2, k=1$ in [17, Equation (24)], the right hand side of the equation gives

$$
\sum_{i=0}^{2}\left(\begin{array}{l}
2 \\
i
\end{array}\right)\left(\frac{1}{2}\right)^{i} q_{4}^{i} q_{3}^{2-i} q_{i+3}=q_{3}^{3}+q_{3} q_{4}^{2}+\frac{1}{4} q_{4}^{2} q_{5}=395,
$$

which contradicts $q_{11}=571$.

If we take $n=2, m=1, k=1$ in [17, Equation (27)], the right hand side of the equation gives

$$
\sum_{i=0}^{2}\left(\begin{array}{l}
2 \\
i
\end{array}\right)\left(\frac{1}{2}\right)^{2-i} q_{3}^{i} q_{2}^{2-i} q_{i+2}=\frac{1}{4} q_{2}^{3}+q_{3}^{2} q_{2}+q_{3}^{2} q_{4}=92
$$

which contradicts $q_{8}=112$.

If we take $n=2, m=1, k=1$ in [17, Equation (30)], the right hand side of the equation gives

$$
\sum_{i=0}^{2}\left(\begin{array}{l}
2 \\
i
\end{array}\right) q_{3}^{i} q_{2}^{2-i} q_{i+3}=q_{2}^{2} q_{3}+2 q_{3} q_{2} q_{4}+q_{3}^{2} q_{5}=207,
$$

which contradicts $q_{9}=153$. 
By considering the identity $\xi(m n+r)=\xi(m n)+\xi(r)-2 \xi(m n) \xi(r)$, we have the following corollary.

Corollary 3.4. For $m, n, r>0$, we have

$$
w_{m n+r}-\left(\frac{b}{a}\right)^{\xi(m)\left(\frac{n+\xi(n)}{2}\right)-\xi(m n) \xi(r)} c^{n} u_{m-1}^{n} w_{r} \equiv 0 \quad\left(\bmod u_{m}\right) .
$$

Now we give a generalization of the Ruggles identity [8] which also generalizes the identities in [17, Theorem $2.2(3-4)]$ and [16, Theorem 1]. Then we give a related binomial identity for the generalized bi-periodic Horadam sequence.

For $n \geq 0$ and $k \geq 1$, the Ruggles identity [8] is given by

$$
F_{n+2 k}=L_{k} F_{n+k}+(-1)^{k+1} F_{n},
$$

where $\left\{F_{n}\right\}$ and $\left\{L_{n}\right\}$ are the Fibonacci and Lucas numbers, respectively. Horadam [4] generalized this result to a general second order recurrence relation

$$
W_{n+2 k}=V_{k} W_{n+k}+(-1)^{k+1} q^{k} W_{n},
$$

where $W_{k}=p W_{k-1}+q W_{k-2}$ with arbitrary initial conditions $W_{0}, W_{1}$ and arbitrary integers $p, q$. The sequence $\left\{V_{k}\right\}$ satisfies the same recurrence relation as the sequence $\left\{W_{k}\right\}$, but it begins with $V_{0}=2, V_{1}=p$.

A generalization of the Ruggles identity can be given in the following lemma.

Lemma 3.5. For integers $n \geq 0$ and $k \geq 1$, we have

$$
w_{n+2 k}=\left(\frac{a}{b}\right)^{\xi(n+1) \xi(k)} v_{k} w_{n+k}-(-c)^{k} w_{n}
$$

where $\left\{w_{n}\right\}$ is the generalized bi-periodic Horadam sequence and $\left\{v_{n}\right\}$ is the generalized bi-periodic Lucas sequence.

Proof. It can be obtained simply by the Binet formula of $\left\{w_{n}\right\}$.

Theorem 3.6. For nonnegative integers $n, r$ and $m$ with $m>1$, we have the following identity:

$$
w_{2 m n+r}=\sum_{i=0}^{n}\left(\begin{array}{c}
n \\
i
\end{array}\right)(-1)^{(m+1)(n-i)}\left(\frac{a}{b}\right)^{\xi(m)\left(\frac{i+\xi(i)}{2}\right)-\xi(i m) \xi(r)} c^{m(n-i)} v_{m}^{i} w_{i m+r} .
$$

Proof. From the Binet formula of $\left\{v_{k}\right\}$ and $\alpha \beta=-a b c$, it is clear to see that

$$
\alpha^{2 m}=a^{\frac{m+\xi(m)}{2}} b^{\frac{m-\xi(m)}{2}} v_{m} \alpha^{m}-(-a b c)^{m} .
$$

By using the binomial theorem, we have

$$
\alpha^{2 m n}=\sum_{i=0}^{n}\left(\begin{array}{c}
n \\
i
\end{array}\right) a^{i \frac{m+\xi(m)}{2}} b^{i \frac{m-\xi(m)}{2}}(-1)^{(m+1)(n-i)}(a b c)^{m(n-i)} v_{m}^{i} \alpha^{i m} .
$$

Similarly, we have

$$
\beta^{2 m n}=\sum_{i=0}^{n}\left(\begin{array}{c}
n \\
i
\end{array}\right) a^{i \frac{m+\xi(m)}{2}} b^{i \frac{m-\xi(m)}{2}}(-1)^{(m+1)(n-i)}(a b c)^{m(n-i)} v_{m}^{i} \beta^{i m} .
$$

Multiplying both sides of the above equalities by $A \alpha^{r}$ and $B \beta^{r}$, respectively, and using the Binet formula of $\left\{w_{n}\right\}$, we get

$$
\begin{aligned}
& \left(A \alpha^{2 m n+r}-B \beta^{2 m n+r}\right) \\
= & a^{-\xi(2 m n+r+1)}(a b)^{\left\lfloor\frac{2 m n+r}{2}\right\rfloor} w_{2 m n+r} \\
= & \sum_{i=0}^{n}\left(\begin{array}{c}
n \\
i
\end{array}\right) a^{i \frac{m+\xi(m)}{2}} b^{i \frac{m-\xi(m)}{2}}(-1)^{(m+1)(n-i)}(a b c)^{m(n-i)} v_{m}^{i}\left(A \alpha^{i m+r}-B \beta^{i m+r}\right) .
\end{aligned}
$$


Thus, again by using the identity $\xi(m n+r)=\xi(m n)+\xi(r)-2 \xi(m n) \xi(r)$, we have

$$
\begin{aligned}
w_{2 m n+r}= & \sum_{i=0}^{n}\left(\begin{array}{c}
n \\
i
\end{array}\right)(-1)^{(m+1)(n-i)} c^{m(n-i)} v_{m}^{i} w_{i m+r} \\
& \times a^{i \frac{m+\xi(m)}{2}} b^{i \frac{m-\xi(m)}{2}}(a b)^{m(n-i)-\left\lfloor\frac{2 m n+r}{2}\right\rfloor+\left\lfloor\frac{i m+r}{2}\right\rfloor} a^{\xi(2 m n+r+1)} a^{-\xi(i m+r+1)}
\end{aligned}
$$

which gives the desired result.

Remark 3.7. Let $c=1$ in Theorem 3.6. Then we have the following results:

(1) For $m=3$ and $r=2 k$, we obtain the identity

$$
w_{6 n+2 k}=\sum_{i=0}^{n}\left(\begin{array}{l}
n \\
i
\end{array}\right)\left(\frac{a}{b}\right)^{\frac{i+\xi(i)}{2}} b^{i}(a b+3)^{i} w_{3 i+2 k} .
$$

(2) For $m=3$ and $r=2 k+1$, we obtain the identity

$$
w_{6 n+2 k+1}=\sum_{i=0}^{n}\left(\begin{array}{l}
n \\
i
\end{array}\right)\left(\frac{a}{b}\right)^{\frac{i-\xi(i)}{2}} b^{i}(a b+3)^{i} w_{3 i+2 k+1} .
$$

We should note that the above results are the corrected results which are given in [17, Equations (14), (17)], respectively. In particular, see the following example which shows that the results in $[17$, Equation $(14),(17)]$ do not hold.

Example 3.8. Consider the same sequence which is given in Example 3.3; that is, $\left\{q_{n}\right\}=$ $\left\{w_{n}(0,1 ; 2,1,1)\right\}$.

If we take $n=2, k=1$ in [17, Equation (14)], the right hand side of the equation gives

$$
\sum_{i=0}^{n}\left(\begin{array}{c}
n \\
i
\end{array}\right) a^{i}(a b+3)^{i} w_{3 i+2 k}=\sum_{i=0}^{2}\left(\begin{array}{l}
2 \\
i
\end{array}\right) 10^{i} q_{3 i+2}=q_{2}+20 q_{5}+100 q_{8}=11422,
$$

which contradicts the result $q_{6 n+2 k}=q_{14}=5822$.

If we take $n=2, k=1$ in [17, Equation (17)], the right hand side of the equation gives

$$
\sum_{i=0}^{n}\left(\begin{array}{c}
n \\
i
\end{array}\right) b^{i}(a b+3)^{i} w_{3 i+2 k+1}=\sum_{i=0}^{2}\left(\begin{array}{c}
2 \\
i
\end{array}\right) 5^{i} q_{3 i+3}=q_{3}+10 q_{6}+25 q_{9}=4128,
$$

which contradicts the result $q_{6 n+2 k+1}=q_{15}=7953$.

Corollary 3.9. For $m, n, r>0$, we have the following identity:

$$
w_{2 m n+r}-(-1)^{(m+1) n} c^{m n} w_{r} \equiv 0 \quad\left(\bmod v_{m}\right) .
$$

Note that for the generalized Fibonacci and Lucas sequences, Corollary 3.9 reduces the identities in [10, 3.3. Corollary].

Lemma 3.10. For $m, r>0$, we have the following identity:

$$
\begin{aligned}
& -(-a b c)^{m+r}+a^{\frac{r+\xi(r)}{2}} b^{\frac{r-\xi(r)}{2}} v_{r}(-a b c)^{m} z^{r}+z^{2(m+r)} \\
= & z^{m+2 r} a^{\frac{m+\xi(m)}{2}} b^{\frac{m-\xi(m)}{2}} v_{m}
\end{aligned}
$$

where $z$ is either $\alpha$ or $\beta$. 
Proof. From the Binet formula of $\left\{v_{n}\right\}$ and $\alpha \beta=-a b c$, it is clear to see that $z^{2 r}=$ $a^{\frac{r+\xi(r)}{2}} b^{\frac{r-\xi(r)}{2}} v_{r} z^{r}-(-a b c)^{r}$. Thus we have

$$
\begin{aligned}
& -(-a b c)^{m+r}+a^{\frac{r+\xi(r)}{2}} b^{\frac{r-\xi(r)}{2}} v_{r}(-a b c)^{m} z^{r}+z^{2(m+r)} \\
= & (-a b c)^{m}\left(a^{\frac{r+\xi(r)}{2}} b^{\frac{r-\xi(r)}{2}} v_{r} z^{r}-(-a b c)^{r}\right)+z^{2(m+r)} \\
= & (-a b c)^{m} z^{2 r}+z^{2(m+r)} \\
= & z^{m+2 r}\left((-a b c)^{m} z^{-m}+z^{m}\right) \\
= & z^{m+2 r}\left(\beta^{m}+\alpha^{m}\right) \\
= & z^{m+2 r} a^{\frac{m+\xi(m)}{2}} b^{\frac{m-\xi(m)}{2}} v_{m} .
\end{aligned}
$$

Theorem 3.11. For $n, m, r>0$, we have the following identity:

$$
\begin{aligned}
& -(-c)^{m+r} w_{n}+(-c)^{m}\left(\frac{a}{b}\right)^{\xi(r) \xi(n+1)} v_{r} w_{r+n}+w_{2(m+r)+n} \\
= & \left(\frac{a}{b}\right)^{\xi(m) \xi(n+1)} v_{m} w_{m+2 r+n} .
\end{aligned}
$$

Proof. From Lemma 3.10, we have

$$
\begin{aligned}
& -(-a b c)^{m+r}+a^{\frac{r+\xi(r)}{2}} b^{\frac{r-\xi(r)}{2}} v_{r}(-a b c)^{m} \alpha^{r}+\alpha^{2(m+r)} \\
= & \alpha^{m+2 r} a^{\frac{m+\xi(m)}{2}} b^{\frac{m-\xi(m)}{2}} v_{m} .
\end{aligned}
$$

Similarly, we have

$$
\begin{aligned}
& -(-a b c)^{m+r}+a^{\frac{r+\xi(r)}{2}} b^{\frac{r-\xi(r)}{2}} v_{r}(-a b c)^{m} \beta^{r}+\beta^{2(m+r)} \\
= & \beta^{m+2 r} a^{\frac{m+\xi(m)}{2}} b^{\frac{m-\xi(m)}{2}} v_{m} .
\end{aligned}
$$

By multiplying both sides of the equations (3.1) and (3.2) by $A \alpha^{n}$ and $B \beta^{n}$, respectively, we get

$$
\begin{aligned}
& -\left(A \alpha^{n}-B \beta^{n}\right)(-a b c)^{m+r} \\
& +a^{\frac{r+\xi(r)}{2}} b^{\frac{r-\xi(r)}{2}} v_{r}(-a b c)^{m}\left(A \alpha^{r+n}-B \beta^{r+n}\right) \\
& +\left(A \alpha^{2(m+r)+n}-B \beta^{2(m+r)+n}\right) \\
= & a^{\frac{m+\xi(m)}{2}} b^{\frac{m-\xi(m)}{2}} v_{m}\left(A \alpha^{m+2 r+n}-B \beta^{m+2 r+n}\right) .
\end{aligned}
$$

Then by using the Binet formula of $\left\{w_{n}\right\}$, we have

$$
\begin{aligned}
& -(a b)^{m+r}(-c)^{m+r} a^{\frac{n+\xi(n)}{2}} b^{\frac{n-\xi(n)}{2}} w_{n} \\
& +(-c)^{m}(a b)^{m+r} a^{\frac{n+\xi(r+n)+\xi(r)}{2}} b^{\frac{n-\xi(r+n)-\xi(r)}{2}} v_{r} w_{r+n} \\
& +(a b)^{m+r} a^{\frac{n+\xi(n)}{2}} b^{\frac{n-\xi(n)}{2}} w_{2(m+r)+n} \\
= & (a b)^{m+r} a^{\frac{n+\xi(m+n)+\xi(m)}{2}} b^{\frac{n-\xi(m+n)-\xi(m)}{2}} v_{m} w_{m+2 r+n} .
\end{aligned}
$$

By considering the identity $\xi(m n+r)=\xi(m n)+\xi(r)-2 \xi(m n) \xi(r)$, we get the desired result.

If we take $r=1, m=2, c=1$ in Theorem 3.11 , we get

$$
(a b+2) w_{n+4}=w_{n}+a^{\xi(n+1)} b^{\xi(n)} w_{n+1}+w_{n+6}
$$


which reduces to the identity

$$
(a b+1) w_{n+4}=w_{n}+a^{\xi(n+1)} b^{\xi(n)} w_{n+1}+a^{\xi(n+1)} b^{\xi(n)} w_{n+5} .
$$

Theorem 3.12. The symbol $\left(\begin{array}{c}n \\ i, j\end{array}\right)$ is defined by $\left(\begin{array}{c}n \\ i, j\end{array}\right):=\frac{n !}{i ! j !(n-i-j) !}$. For $n, m, r, d>0$, we have the following identities:

$$
\begin{aligned}
w_{(m+2 r) n+d}= & v_{m}^{-n} \sum_{i+j+s=n}\left(\begin{array}{c}
n \\
i, j
\end{array}\right)(-1)^{s}(-c)^{m j+(m+r) s} v_{r}^{j} w_{2(m+r) i+r j+d} \\
& \times\left(\frac{a}{b}\right)^{\xi(r) \frac{j+\xi(j)}{2}-\xi(m) \frac{n+\xi(n)}{2}-\xi(r j) \xi(d)+\xi(m n) \xi(d)}
\end{aligned}
$$

and

$$
\begin{aligned}
w_{2(m+r) n+d}= & \sum_{i+j+s=n}\left(\begin{array}{c}
n \\
i, j
\end{array}\right)(-1)^{j}(-c)^{s(m+r)+m j} v_{m}^{i} v_{r}^{j} w_{(m+2 r) i+r j+d} \\
& \times\left(\frac{a}{b}\right)^{\xi(m) \frac{i+\xi(i)}{2}+\xi(r) \frac{j+\xi(j)}{2}-\xi(m i) \xi(r j)-\xi(m i+r j) \xi(d)} .
\end{aligned}
$$

Proof. By using Lemma 3.10 and the multinomial theorem, we obtain the following identities:

$$
\begin{aligned}
& a^{n \frac{m+\xi(m)}{2}} b^{n \frac{m-\xi(m)}{2}} v_{m}^{n} z^{(m+2 r) n} \\
= & \sum_{i+j+s=n}\left(\begin{array}{c}
n \\
i, j
\end{array}\right)(-1)^{s}(-a b c)^{s(m+r)+m j} a^{j \frac{r+\xi(r)}{2}} b^{j \frac{r-\xi(r)}{2}} v_{r}^{j} z^{2(m+r) i+r j}
\end{aligned}
$$

and

$$
=\sum_{i+j+s=n}\left(\begin{array}{c}
n \\
i, j
\end{array}\right)(-1)^{j}(-a b c)^{s(m+r)+m j} a^{\frac{i m+i \xi(m)+j r+j \xi(r)}{2}} b^{\frac{i m-i \xi(m)+j r-j \xi(r)}{2}} v_{m}^{i} v_{r}^{j} z^{(m+2 r) i+r j} .
$$

By multiplying both sides in the preceding equalities by $z^{d}$ and using the Binet formula of $\left\{w_{n}\right\}$, we have (3.3) and (3.4), respectively.

From (3.4), by using the decomposition

$$
\sum_{i+j+s=n}=\sum_{i+j+s=n, i=0}+\sum_{i+j+s=n, i \neq 0}
$$

and Theorem 3.6, we get the following corollary.

Corollary 3.13. For $n, m, r, d>0$, we have

$$
w_{2(m+r) n+d}-(-1)^{n(m+1)} c^{m n} w_{2 r n+d} \equiv 0 \quad\left(\bmod v_{m}\right) .
$$

Acknowledgment. The first author is grateful to Dr. Mohamed Salim for the arrangement of her visit to United Arab Emirates University (UAEU) in February 2019. It is supported by UAEU UPAR Grant G00002599 (Fund No. 31S314).

\section{References}

[1] G. Bilgici, Two generalizations of Lucas sequence, Appl. Math. Comput. 245, 526538,2014

[2] L. Carlitz and H.H. Ferns, Some Fibonacci and Lucas Identities, Fibonacci Quart. 8 (1), 61-73, 1970. 
[3] M. Edson and O. Yayenie, A new generalizations of Fibonacci sequences and extended Binet's Formula, Integers, 9, 639-654, 2009.

[4] A.F. Horadam, Basic Properties of a Certain Generalized Sequence of Numbers, Fibonacci Quart. 3 (3), 161-76, 1965.

[5] L.C. Hsu and M.S. Jiang, A kind of invertible graphical process for finding reciprocal formulas with applications, Acta Sci. Nat. Univ. Jilinensis, 4, 43-55, 1980.

[6] E. Kilic and E. Tan, More General Identities Involving The Terms Of $\{W n(a, b ; p, q)\}$, Ars Comb. 93, 459-461, 2009.

[7] D. Panario, M. Sahin and Q. Wang, A family of Fibonacci-like conditional sequences, Integers, 13 (A78), 2013.

[8] I.D. Ruggles, Some Fibonacci results using Fibonacci-type sequences, Fibonacci Quart. 1 (2), 75-80, 1963.

[9] M. Sahin, The Gelin-Cesaro identity in some conditional sequences, Hacet. J. Math. Stat. 40 (6), 855-861, 2011.

[10] Z. Siar and R. Keskin, Some new identities concerning generalized Fibonacci and Lucas numbers, Hacet. J. Math. Stat. 42 (3), 211-222, 2013.

[11] E. Tan, On bi-periodic Fibonacci and Lucas numbers by matrix method, Ars Combin. 133, 107-113, 2017.

[12] E. Tan, Some properties of the bi-periodic Horadam sequences, Notes Number Theory Discrete Math. 23 (4), 56-65, 2017.

[13] E. Tan and A.B. Ekin, Bi-Periodic Incomplete Lucas Sequences, Ars Combin. 123, 371-380, 2015.

[14] E. Tan and A.B. Ekin, Some Identities On Conditional Sequences By Using Matrix Method, Miskolc Math. Notes, 18 (1), 469-477, 2017.

[15] E. Tan and H.H. Leung, Some basic properties of the generalized bi-periodic Fibonacci and Lucas sequences, Adv. Difference Equ. 2020 (26), 2020.

[16] O. Yayenie, A note on generalized Fibonacci sequence, Appl. Math. Comput. 217, 5603-5611, 2011.

[17] J. Yang and Z. Zhang, Some identities of the generalized Fibonacci and Lucas sequences, Appl. Math. Comput. 339, 451-458, 2018.

[18] Z. Zhang, Some properties of the generalized fibonacci sequences $c_{n}=c_{n-1}+c_{n-2}+r$, Fibonacci Quart. 35 (2), 169-171, 1997.

[19] Z. Zhang and M. Liu, Generalizations of some identities involving generalized secondorder integer sequences, Fibonacci Quart. 36 (4), 327-328, 1998. 